\title{
ON A WAVE EQUATION WITH A BOUNDARY CONDITION ASSOCIATED WITH CAPILLARY WAVES*
}

\author{
JONG UHN $\mathrm{KIM}^{\dagger}$
}

\begin{abstract}
This paper discusses an initial-boundary value problem for a wave equation with a nonstandard boundary condition associated with linear capillary waves on the surface of a compressible liquid. We prove the well-posedness of this problem. Our main technical device is the Fourier transform.
\end{abstract}

Key words. wave equation, initial-boundary value problem, capillary waves

AMS subject classifications. 35B30, 35D05, 35L05

PII. S003614109732821X

Introduction. In this paper we discuss the following initial-boundary value problem for a wave equation:

$$
\begin{gathered}
u_{t t}-\Delta u=0 \quad \text { for } t \in(0, T),(x, y, z) \in R_{+}^{3}, \\
\mathcal{B} u(t, x, y, 0)=0 \quad \text { for } t \in(0, T),(x, y) \in R^{2}, \\
u(0, x, y, z)=u_{0}(x, y, z), \quad u_{t}(0, x, y, z)=u_{1}(x, y, z) \quad \text { for }(x, y, z) \in R_{+}^{3},
\end{gathered}
$$

where $T>0$ is arbitrarily given, $R_{+}^{3}=\left\{(x, y, z):(x, y) \in R^{2}, z>0\right\}, \Delta$ is the Laplacian in $(x, y, z)$, and the operator $\mathcal{B}$ is defined by

$$
\mathcal{B} u=\Delta u+\frac{\partial}{\partial z}\left(u_{x x}+u_{y y}\right)
$$

This nonstandard boundary condition arises in the linearized model of capillary waves. Let a compressible liquid occupy the half-space $R_{+}^{3}$, and let $u$ denote its velocity potential. When the effects of gravity are neglected, $u$ satisfies the following acoustic equation within the liquid:

$$
u_{t t}-c^{2} \Delta u=0
$$

where $c$ is the speed of sound. We describe the free surface by $z=\zeta(t, x, y)$. By the Laplace formula (see Landau and Lifshitz [5]), we have

$$
p-p_{0}=\alpha\left(\zeta_{x x}+\zeta_{y y}\right),
$$

where $\alpha>0$ is the surface-tension coefficient, $p$ is the pressure in the liquid near the surface, and $p_{0}$ is the constant external pressure. On the other hand, $u$ is related to the pressure by

$$
p-p_{0}=-\rho_{0} \frac{\partial u}{\partial t}
$$

\footnotetext{
${ }^{*}$ Received by the editors October 3, 1997; accepted for publication (in revised form) March 4, 1998; published electronically September 29, 1998.

http://www.siam.org/journals/sima/30-1/32821.html

${ }^{\dagger}$ Department of Mathematics, Virginia Tech, Blacksburg, VA 24061-0123 (kim@math.vt.edu).
} 
where $\rho_{0}$ is the constant equilibrium density and gravity has been neglected. On the surface, it holds that

$$
\frac{\partial u}{\partial z}=\frac{\partial \zeta}{\partial t} \quad \text { at } z=0 .
$$

Combining (0.5)-(0.8), we have

$$
\Delta u+\frac{\alpha}{\rho_{0} c^{2}} \frac{\partial}{\partial z}\left(u_{x x}+u_{y y}\right)=0 \quad \text { at } z=0 .
$$

By rescaling the variables, we arrive at $(0.1)$ and (0.2).

For the Dirichlet boundary condition $u=0$, or the Neumann boundary condition $\frac{\partial u}{\partial z}=0$ at $z=0$, a complete result on the well-posedness of the initial-boundary value problem is well known. The purpose of this work is to establish a similar result in the $L^{2}$ Sobolev spaces with the boundary condition (0.2). Bondi [2] constructed a fundamental solution of (0.1)-(0.2) and studied an interesting phenomenon of wave propagation along the surface with infinite speed. His result was also discussed by Duff [3]. Here our issue is to formulate the well-posedness of the initial-boundary value problem (0.1)-(0.3) as closely as possible to that of the standard initial-boundary value problems. One could use fundamental solutions to represent solutions. However, even for the pure Cauchy problem in the whole space, the explicit formula of the fundamental solution is not a good device to obtain the basic $L^{2}$ energy estimates, which trivially follow either by a multiplier technique or by the Fourier transform. In particular, it is not at all clear whether the fundamental solution obtained in [2] can be used to obtain estimates of solutions in the function classes of $L^{2}$ setting. Here we rely on the well-known theory of the Cauchy problem in the whole space. This involves a suitable extension of the given data to the whole space, where various compatibility conditions are naturally incorporated. The main tool is the Fourier transform. When the boundary operator consists of only odd (or only even) derivatives in the normal coordinate variable, this is a standard procedure. The novelty of the above problem is that the boundary operator is hybrid, and a simple reflection method does not work. So the main difficulty lies in handling the boundary condition. The boundary $z=0$ is noncharacteristic, and the trace of any derivative of the solution is well-defined due to partial hypoellipticity; see Hörmander [4]. But we need some regularity condition for the uniqueness of the solution, which is justified by a simple example presented in the next section. Our starting point is the following observation made by Duff [3]. Since $\mathcal{B}$ is a differential operator with constant coefficients, $\mathcal{B} u$ satisfies the wave equation if $u$ does. Consequently, $\mathcal{B} u$ is a solution of the initial-boundary value problem with the initial data $\left(\mathcal{B} u_{0}, \mathcal{B} u_{1}\right)$ and the Dirichlet boundary condition. However, it is only a weak solution unless $u$ itself is sufficiently smooth. For this, we provide in the next section a definition of a weak solution which is suitable in our function classes such that each solution in the natural energy space is included. As the initial data are more regular with suitable compatibility conditions at the boundary $z=0$, the regularity of the solution improves accordingly.

Following the notation for function classes in Lions and Magenes [6], we state the main result as follows.

Theorem 0.1. Let $u_{0} \in H^{2}\left(R_{+}^{3}\right)$ and $u_{1} \in H^{1}\left(R_{+}^{3}\right)$ with $\frac{\partial u_{0}}{\partial z}(x, y, 0) \in H^{1}\left(R^{2}\right)$. Then, there is a unique solution $u \in C\left([0, T] ; H^{2}\left(R_{+}^{3}\right)\right) \cap C^{1}\left([0, T] ; H^{1}\left(R_{+}^{3}\right)\right)$ of (0.1)-(0.3) such that $\mathcal{B} u$ is a solution of $(1.9)$ below with the initial data $\left(\mathcal{B} u_{0}, \mathcal{B} u_{1}\right)$. 
Furthermore, for each $t \in[0, T]$ the solution satisfies

$$
\begin{aligned}
& \|u(t, \cdot)\|_{H^{2}\left(R_{+}^{3}\right)}+\left\|u_{t}(t, \cdot)\right\|_{H^{1}\left(R_{+}^{3}\right)} \\
& \quad \leq M(T)\left(\left\|u_{0}\right\|_{H^{2}\left(R_{+}^{3}\right)}+\left\|u_{1}\right\|_{H^{1}\left(R_{+}^{3}\right)}+\left\|\frac{\partial u_{0}}{\partial z}(\cdot, 0)\right\|_{H^{1}\left(R^{2}\right)}\right),
\end{aligned}
$$

where $M(T)$ is a positive constant depending on $T$.

We can also obtain more regular solutions.

Corollary 0.2. If we suppose that $u_{0} \in H^{3}\left(R_{+}^{3}\right)$ with $\mathcal{B} u_{0}=0$ at $z=0$ and that $u_{1} \in H^{2}\left(R_{+}^{3}\right)$ with $\frac{\partial u_{1}}{\partial z}(\cdot, 0) \in H^{1}\left(R^{2}\right)$, the above solution belongs to $C\left([0, T] ; H^{3}\left(R_{+}^{3}\right)\right) \cap C^{1}\left([0, T] ; H^{2}\left(R_{+}^{3}\right)\right)$, and it holds that for all $t \in[0, T]$,

$$
\begin{aligned}
& \|u(t, \cdot)\|_{H^{3}\left(R_{+}^{3}\right)}+\left\|u_{t}(t, \cdot)\right\|_{H^{2}\left(R_{+}^{3}\right)} \\
& \quad \leq M(T)\left(\left\|u_{0}\right\|_{H^{3}\left(R_{+}^{3}\right)}+\left\|u_{1}\right\|_{H^{2}\left(R_{+}^{3}\right)}+\left\|\frac{\partial u_{1}}{\partial z}(\cdot, 0)\right\|_{H^{1}\left(R^{2}\right)}\right)
\end{aligned}
$$

for some positive constant $M(T)$.

Corollary 0.3. Let $m \geq 1$ be an integer. Suppose that $u_{0} \in H^{2 m+2}\left(R_{+}^{3}\right), \mathcal{B} u_{0}=$ $0, \Delta \mathcal{B} u_{0}=0, \ldots, \Delta^{m-1} \mathcal{B} u_{0}=0$ at $z=0,\left(\frac{\partial}{\partial z}\right)^{2 m+1} u_{0}(\cdot, 0) \in H^{1}\left(R^{2}\right)$ and that $u_{1} \in H^{2 m+1}\left(R_{+}^{3}\right), \mathcal{B} u_{1}=0, \Delta \mathcal{B} u_{1}=0, \ldots, \Delta^{m-1} \mathcal{B} u_{1}=0$ at $z=0$. Then, the above solution belongs to $C\left([0, T] ; H^{2 m+2}\left(R_{+}^{3}\right)\right) \cap C^{1}\left([0, T] ; H^{2 m+1}\left(R_{+}^{3}\right)\right)$ and satisfies for all $t \in[0, T]$,

$$
\begin{aligned}
& \|u(t, \cdot)\|_{H^{2 m+2}\left(R_{+}^{3}\right)}+\left\|u_{t}(t, \cdot)\right\|_{H^{2 m+1}\left(R_{+}^{3}\right)} \\
& \quad \leq M(T, m)\left(\left\|u_{0}\right\|_{H^{2 m+2}\left(R_{+}^{3}\right)}+\left\|u_{1}\right\|_{H^{2 m+1}\left(R_{+}^{3}\right)}+\left\|\frac{\partial^{2 m+1} u_{0}}{\partial z^{2 m+1}(\cdot, 0)}\right\|_{H^{1}\left(R^{2}\right)}\right),
\end{aligned}
$$

where $M(T, m)$ is a positive constant depending on $T$ and $m$.

Corollary 0.4. Let $m \geq 1$ be an integer. Suppose that $u_{0} \in H^{2 m+3}\left(R_{+}^{3}\right), \mathcal{B} u_{0}=$ $0, \Delta \mathcal{B} u_{0}=0, \ldots, \Delta^{m} \mathcal{B} u_{0}=0$ at $z=0$ and that $u_{1} \in H^{2 m+2}\left(R_{+}^{3}\right), \mathcal{B} u_{1}=$ $0, \Delta \mathcal{B} u_{1}=0, \ldots, \Delta^{m-1} \mathcal{B} u_{1}=0$ at $z=0,\left(\frac{\partial}{\partial z}\right)^{2 m+1} u_{1}(\cdot, 0) \in H^{1}\left(R^{2}\right)$. Then, the above solution belongs to $C\left([0, T] ; H^{2 m+3}\left(R_{+}^{3}\right)\right) \cap C^{1}\left([0, T] ; H^{2 m+2}\left(R_{+}^{3}\right)\right)$ and satisfies for all $t \in[0, T]$,

$$
\begin{aligned}
& \|u(t, \cdot)\|_{H^{2 m+3}\left(R_{+}^{3}\right)}+\left\|u_{t}(t, \cdot)\right\|_{H^{2 m+2}\left(R_{+}^{3}\right)} \\
& \quad \leq M(T, m)\left(\left\|u_{0}\right\|_{H^{2 m+3}\left(R_{+}^{3}\right)}+\left\|u_{1}\right\|_{H^{2 m+2}\left(R_{+}^{3}\right)}+\left\|\frac{\partial^{2 m+1} u_{1}}{\partial z^{2 m+1}(\cdot, 0)}\right\|_{H^{1}\left(R^{2}\right)}\right) .
\end{aligned}
$$

If we assume that the support of the initial data is contained in $R_{+}^{3}$, then the result is essentially the same as that for the standard boundary conditions.

COROLlary 0.5. Let $m$ be a nonnegative integer. Let $u_{0} \in H^{m+2}\left(R_{+}^{3}\right)$ and $u_{1} \in H^{m+1}\left(R_{+}^{3}\right)$ such that $\operatorname{supp} u_{0} \cup \operatorname{supp} u_{1} \subset R_{+}^{3}$. Then, the above solution belongs to $C\left([0, T] ; H^{m+2}\left(R_{+}^{3}\right)\right) \cap C^{1}\left([0, T] ; H^{m+1}\left(R_{+}^{3}\right)\right)$ and satisfies for each $t \in[0, T]$,

$$
\begin{aligned}
& \|u(t, \cdot)\|_{H^{m+2}\left(R_{+}^{3}\right)}+\left\|u_{t}(t, \cdot)\right\|_{H^{m+1}\left(R_{+}^{3}\right)} \\
& \quad \leq M(T, m)\left(\left\|u_{0}\right\|_{H^{m+2}\left(R_{+}^{3}\right)}+\left\|u_{1}\right\|_{H^{m+1}\left(R_{+}^{3}\right)}\right) .
\end{aligned}
$$

We will prove these results in the next sections. 
1. Preliminaries. We first review some facts about the Cauchy problem for a wave equation in $R^{3}$, which is formulated as follows:

$$
\left\{\begin{array}{l}
u_{t t}-\Delta u=0 \quad \text { in }(0, T) \times R^{3} \\
u(0, x, y, z)=u_{0}(x, y, z) \quad \text { in } R^{3} \\
u_{t}(0, x, y, z)=u_{1}(x, y, z) \quad \text { in } R^{3}
\end{array}\right.
$$

where $\Delta$ is the Laplacian in $(x, y, z) \in R^{3}$, and $0<T<\infty$ is arbitrarily given.

LEMmA 1.1. Let $u_{0} \in H^{s}\left(R^{3}\right)$, and $u_{1} \in H^{s-1}\left(R^{3}\right)$, for some $s \in R$. Then, there is a unique solution of the Cauchy problem $(1.1)$ in $C\left([0, T] ; H^{s}\left(R^{3}\right)\right) \cap C^{1}([0, T]$; $\left.H^{s-1}\left(R^{3}\right)\right)$, and it holds that

$$
\|u(t, \cdot)\|_{H^{s}\left(R^{3}\right)}+\left\|u_{t}(t, \cdot)\right\|_{H^{s-1}\left(R^{3}\right)} \leq M(T)\left(\left\|u_{0}\right\|_{H^{s}\left(R^{3}\right)}+\left\|u_{1}\right\|_{H^{s-1}\left(R^{3}\right)}\right)
$$

for all $t \in[0, T]$ with some positive constant $M(T)$ independent of $u_{0}$ and $u_{1}$.

LEMMA 1.2. In the same setting as above, assume that supp $u_{0} \cup \operatorname{supp} u_{1}$ $\subset\{(x, y, z): z<-L\}$ for some $L \in R$. Then, the support of the solution for $t \geq 0$ is contained in $\{(t, x, y, z): t>z+L\}$.

Next we define a function space $\mathcal{X}$ by

$$
\begin{aligned}
\mathcal{X}= & H^{-1}\left(R_{z} ; H^{-1}\left(R^{2}\right)\right)+L^{2}\left(R_{z} ; H^{-2}\left(R^{2}\right)\right) \\
& =\left\{f_{1}+f_{2}: f_{1}(x, y, z) \in H^{-1}\left(R_{z} ; H^{-1}\left(R^{2}\right)\right), \quad f_{2}(x, y, z) \in L^{2}\left(R_{z} ; H^{-2}\left(R^{2}\right)\right)\right\}
\end{aligned}
$$

equipped with the norm

$$
\|f\|_{\mathcal{X}}=\inf _{f=f_{1}+f_{2}}\left(\left\|f_{1}\right\|_{H^{-1}\left(R_{z} ; H^{-1}\left(R^{2}\right)\right)}+\left\|f_{2}\right\|_{L^{2}\left(R_{z} ; H^{-2}\left(R^{2}\right)\right)}\right),
$$

where $R_{z}$ is for the $z$ variable. Then, $\mathcal{X}$ is the dual of $H^{1}\left(R_{z} ; H^{1}\left(R^{2}\right)\right) \cap L^{2}\left(R_{z} ; H^{2}\left(R^{2}\right)\right)$. For this, see Bergh and Löfström [1]. We also need the following fact.

LEMMA 1.3. The operator $\left(1-\Delta_{x, y}\right)^{1 / 2}$ is an isomorphism from $H^{-1}\left(R^{3}\right)$ onto $\mathcal{X}$. Here, $\Delta_{x, y}$ is the Laplacian in $(x, y)$.

Proof. Choose any $f \in H^{-1}\left(R^{3}\right)$, and set

$$
\hat{f}_{1}(\xi)=\frac{\left|\xi_{3}\right|}{1+\left|\xi_{1}\right|+\left|\xi_{2}\right|+\left|\xi_{3}\right|} \hat{f}(\xi)
$$

and

$$
\hat{f}_{2}(\xi)=\frac{1+\left|\xi_{1}\right|+\left|\xi_{2}\right|}{1+\left|\xi_{1}\right|+\left|\xi_{2}\right|+\left|\xi_{3}\right|} \hat{f}(\xi)
$$

where $\hat{f}(\xi)$ is the Fourier transform of $f$ and $\xi=\left(\xi_{1}, \xi_{2}, \xi_{3}\right)$ is the dual variable of $(x, y, z)$. It is apparent that $f=f_{1}+f_{2}$ and

$$
\left\|\left(1-\Delta_{x, y}\right)^{1 / 2} f_{1}\right\|_{H^{-1}\left(R_{z} ; H^{-1}\left(R^{2}\right)\right)} \leq M\|f\|_{H^{-1}\left(R^{3}\right)}
$$

and

$$
\left\|\left(1-\Delta_{x, y}\right)^{1 / 2} f_{2}\right\|_{L^{2}\left(R_{z} ; H^{-2}\left(R^{2}\right)\right)} \leq M\|f\|_{H^{-1}\left(R^{3}\right)}
$$

for some positive constant $M$ independent of $f$. In the meantime, $H^{-1}\left(R_{z} ; L^{2}\left(R^{2}\right)\right)$ and $L^{2}\left(R_{z} ; H^{-1}\left(R^{2}\right)\right)$ are continuously embedded into $H^{-1}\left(R^{3}\right)$. Hence, $\left(1-\Delta_{x, y}\right)^{-1 / 2}$ is continuous from $\mathcal{X}$ into $H^{-1}\left(R^{3}\right)$. 
Lemma 1.4. Suppose that $u_{0} \in L^{2}\left(R_{z} ; H^{-1}\left(R^{2}\right)\right)$ and $u_{1} \in \mathcal{X}$. Then, there is a unique solution $u$ of $(1.1)$ in $C\left([0, T] ; L^{2}\left(R_{z} ; H^{-1}\left(R^{2}\right)\right)\right) \cap C^{1}([0, T] ; \mathcal{X})$. We also have

$$
\|u(t, \cdot)\|_{L^{2}\left(R_{z} ; H^{-1}\left(R^{2}\right)\right)}+\left\|u_{t}(t, \cdot)\right\|_{\mathcal{X}} \leq M(T)\left(\left\|u_{0}\right\|_{L^{2}\left(R_{z} ; H^{-1}\left(R^{2}\right)\right)}+\left\|u_{1}\right\|_{\mathcal{X}}\right)
$$

for all $t \in[0, T]$, where $M(T)$ is a positive constant independent of $u_{0}$ and $u_{1}$.

Proof. Let us set

$$
v_{0}=\left(1-\Delta_{x, y}\right)^{-1 / 2} u_{0}, \quad v_{1}=\left(1-\Delta_{x, y}\right)^{-1 / 2} u_{1},
$$

and consider the Cauchy problem (1.1) with $\left(u_{0}, u_{1}\right)$ replaced by $\left(v_{0}, v_{1}\right)$. Since $v_{0} \in$ $L^{2}\left(R^{3}\right)$ and $v_{1} \in H^{-1}\left(R^{3}\right)$, it follows from Lemma 1.1 that there is a unique solution $v$ in $C\left([0, T] ; L^{2}\left(R^{3}\right)\right) \cap C^{1}\left([0, T] ; H^{-1}\left(R^{3}\right)\right)$. We then set

$$
u=\left(1-\Delta_{x, y}\right)^{1 / 2} v .
$$

By virtue of Lemmas 1.1 and 1.3, we find that $u$ is the unique solution of (1.1) in $C\left([0, T] ; L^{2}\left(R_{z} ; H^{-1}\left(R^{2}\right)\right)\right) \cap C^{1}([0, T] ; \mathcal{X})$ and that $(1.6)$ is satisfied.

We now consider an initial-boundary value problem in a half-space:

$$
\left\{\begin{array}{l}
u_{t t}-\Delta u=0 \quad \text { in } \quad(0, T) \times R_{+}^{3} \\
u(t, x, y, 0)=0 \quad \text { in } \quad(0, T) \times R^{2} \\
u(0, x, y, z)=u_{0}(x, y, z) \quad \text { in } R_{+}^{3} \\
u_{t}(0, x, y, z)=u_{1}(x, y, z) \quad \text { in } R_{+}^{3} .
\end{array}\right.
$$

We define a function space $\mathcal{X}_{1}$ by

$$
\mathcal{X}_{1}=\left\{f_{1}+f_{2}: f_{1} \in H^{-1}\left((0, \infty) ; H^{-1}\left(R^{2}\right)\right), f_{2} \in L^{2}\left(0, \infty ; H^{-2}\left(R^{2}\right)\right)\right\}
$$

equipped with the norm

$$
\|f\|_{\mathcal{X}_{1}}=\inf _{f=f_{1}+f_{2}}\left(\left\|f_{1}\right\|_{H^{-1}\left((0, \infty) ; H^{-1}\left(R^{2}\right)\right)}+\left\|f_{2}\right\|_{L^{2}\left(0, \infty ; H^{-2}\left(R^{2}\right)\right)}\right),
$$

so that $\mathcal{X}_{1}$ is the dual of $H_{0}^{1}\left((0, \infty) ; H^{1}\left(R^{2}\right)\right) \cap L^{2}\left(0, \infty ; H^{2}\left(R^{2}\right)\right)$. We then adopt the following definition of a solution of (1.9).

Definition 1.5. Let $u_{0} \in L^{2}\left(0, \infty ; H^{-1}\left(R^{2}\right)\right)$ and $u_{1} \in \mathcal{X}_{1}$. We say that a function $u \in C\left([0, T] ; L^{2}\left(0, \infty ; H^{-1}\left(R^{2}\right)\right)\right) \cap C^{1}\left([0, T] ; \mathcal{X}_{1}\right)$ is a solution of $(1.9)$ if it holds that

$$
\begin{aligned}
-\left\langle u_{1}, \phi(0, x, y, z)\right\rangle_{1} & +\left\langle u_{0}, \phi_{t}(0, x, y, z)\right\rangle_{2} \\
& +\int_{0}^{\infty} \int_{0}^{T}\left\langle u, \phi_{t t}-\Delta \phi\right\rangle_{3} d t d z=0
\end{aligned}
$$

for every $\phi \in C^{2}\left([0, T] ; H^{2}\left(R_{+}^{3}\right)\right)$ such that

$$
\left\{\begin{array}{l}
\phi(t, x, y, 0)=0 \quad \text { in } \quad[0, T] \times R^{2}, \\
\phi(T, x, y, z)=0, \quad \phi_{t}(T, x, y, z)=0 \quad \text { in } \quad R_{+}^{3} .
\end{array}\right.
$$

Here, the bracket $\langle\cdot, \cdot\rangle_{1}$ is the duality pairing between $\mathcal{X}_{1}$ and $H_{0}^{1}\left((0, \infty) ; H^{1}\left(R^{2}\right)\right) \cap$ $L^{2}\left(0, \infty ; H^{2}\left(R^{2}\right)\right),\langle\cdot, \cdot\rangle_{2}$ is the duality pairing between $L^{2}\left(0, \infty ; H^{-1}\left(R^{2}\right)\right)$ and $L^{2}\left(0, \infty ; H^{1}\left(R^{2}\right)\right)$, and $\langle\cdot, \cdot\rangle_{3}$ is the duality pairing between $H^{-1}\left(R^{2}\right)$ and $H^{1}\left(R^{2}\right)$. 
First we note that the solution in the natural energy space $C\left([0, T] ; H_{0}^{1}\left(R_{+}^{3}\right)\right) \cap$ $C^{1}\left([0, T] ; L^{2}\left(R_{+}^{3}\right)\right)$ satisfies the above condition (1.11). We will show that the condition (1.11) implies the boundary condition at $z=0$. Choose any $\psi \in C_{0}^{\infty}((0, T) \times$ $R^{2}$ ), and set

$$
\begin{gathered}
J(z)=\int_{0}^{T}\langle u(t, x, y, z), \psi(t, x, y)\rangle_{3} d t, \\
I(z)=\int_{0}^{T}\left\langle u(t, x, y, z), \psi_{t t}-\Delta_{x, y} \psi\right\rangle_{3} d t .
\end{gathered}
$$

Then, $J(z)$ and $I(z)$ belong to $L^{2}((0, \infty))$, and it holds that

$$
\frac{d^{2} J}{d z^{2}}(z)=I(z), \quad \text { in } \quad \mathcal{D}^{\prime}((0, \infty))
$$

since (1.11) implies that $u$ satisfies the wave equation in $\mathcal{D}^{\prime}\left((0, T) \times R_{+}^{3}\right)$. Thus, $J(z) \in H^{2}((0, \infty)) \subset C^{1}([0, T])$. Next choose $p(z) \in C_{0}^{\infty}([0, \infty))$ with $p(0)=0$, and $\frac{d p}{d z}(0)=1$. It follows from (1.13) that

$$
\int_{0}^{\infty} \frac{d^{2} J}{d z^{2}}(z) p(z) d z=\int_{0}^{\infty} I(z) p(z) d z
$$

But we have

$$
\int_{0}^{\infty} \frac{d^{2} J}{d z^{2}} p(z) d z=J(0)+\int_{0}^{\infty} J(z) \frac{d^{2} p}{d z^{2}}(z) d z .
$$

By (1.11), we find that

$$
\begin{gathered}
0=\int_{0}^{\infty} \int_{0}^{T}\left\langle u, \psi_{t t}-\Delta_{x, y} \psi\right\rangle_{3} p(z) d t d z-\int_{0}^{\infty} \int_{0}^{T}\langle u, \psi\rangle_{3} \frac{d^{2} p}{d z^{2}}(z) d t d z \\
=\int_{0}^{\infty} I(z) p(z) d z-\int_{0}^{\infty} J(z) \frac{d^{2} p}{d z^{2}}(z) d z
\end{gathered}
$$

It follows from (1.14)-(1.16) that $J(0)=0$, which means that $u$ satisfies the boundary condition. Next we show that (1.11) implies that the initial conditions are satisfied. Choose an arbitrary function $\theta(x, y, z) \in C_{0}^{\infty}\left(R_{+}^{3}\right)$, and set

$$
\begin{gathered}
K(t)=\int_{0}^{\infty}\langle u(t, x, y, z), \theta(x, y, z)\rangle_{3} d z, \\
L(t)=\int_{0}^{\infty}\langle u(t, x, y, z), \Delta \theta(x, y, z)\rangle_{3} d z .
\end{gathered}
$$

Then, $K(t)$ and $L(t)$ are continuous in $t \in[0, T]$, and

$$
\frac{d^{2} K}{d t^{2}}(t)=L(t) \quad \text { holds in } \quad \mathcal{D}^{\prime}((0, T)),
$$


which yields $K(t) \in C^{2}([0, T])$. Next choose a function $q(t) \in C^{\infty}([0, T])$ such that $q(T)=q_{t}(T)=0, \quad q(0)=1$, and $q_{t}(0)=0$. It is obvious that

$$
\int_{0}^{T} \frac{d^{2} K}{d t^{2}}(t) q(t) d t=\int_{0}^{T} L(t) q(t) d t
$$

and thus,

$$
-\frac{d K}{d t}(0)+\int_{0}^{T} K(t) \frac{d^{2} q}{d t^{2}}(t) d t=\int_{0}^{T} L(t) q(t) d t .
$$

By virtue of (1.11), it holds that

$$
\begin{aligned}
0= & -\left\langle u_{1}, \theta(x, y, z)\right\rangle_{1}+\int_{0}^{\infty} \int_{0}^{T}\left\langle u, q_{t t} \theta-q \Delta \theta\right\rangle_{3} d t d z \\
& =-\left\langle u_{1}, \theta(x, y, z)\right\rangle_{1}+\int_{0}^{T} K(t) q_{t t}(t) d t-\int_{0}^{T} L(t) q(t) d t .
\end{aligned}
$$

By combining (1.19) and (1.20), we have

$$
\left\langle u_{1}, \theta(x, y, z)\right\rangle_{1}=\frac{d K}{d t}(0)=\left\langle u_{t}(0, x, y, z), \theta(x, y, z)\right\rangle_{1} .
$$

By choosing a different $q(t)$, it also holds that

$$
\left\langle u_{0}, \theta(x, y, z)\right\rangle_{2}=\langle u(0, x, y, z), \theta(x, y, z)\rangle_{2} .
$$

Hence, $u$ satisfies the initial conditions.

Proposition 1.6. Let $u_{0} \in L^{2}\left(0, \infty ; H^{-1}\left(R^{2}\right)\right)$ and $u_{1} \in \mathcal{X}_{1}$. Then, there is a unique solution of $(1.9)$ in $C\left([0, T] ; L^{2}\left(0, \infty ; H^{-1}\left(R^{2}\right)\right)\right) \cap C^{1}\left([0, T] ; \mathcal{X}_{1}\right)$. and

Proof. We can choose a sequence of functions $\left\{u_{0}^{n}\right\}_{n=1}^{\infty}$ such that $u_{0}^{n} \in C_{0}^{\infty}\left(R_{+}^{3}\right)$

$$
u_{0}^{n} \rightarrow u_{0} \quad \text { in } \quad L^{2}\left(0, \infty ; H^{-1}\left(R^{2}\right)\right) .
$$

Since $u_{1} \in \mathcal{X}_{1}$, we can write

$$
u_{1}=\frac{d f}{d z}+g
$$

for some $f \in L^{2}\left(0, \infty ; H^{-1}\left(R^{2}\right)\right)$ and $g \in L^{2}\left(0, \infty ; H^{-2}\left(R^{2}\right)\right)$. Let $\left\{f_{n}\right\}_{n=1}^{\infty}$ and $\left\{g_{n}\right\}_{n=1}^{\infty}$ be sequences in $C_{0}^{\infty}\left(R_{+}^{3}\right)$ such that

$$
\begin{aligned}
& f_{n} \rightarrow f \quad \text { in } \quad L^{2}\left(0, \infty ; H^{-1}\left(R^{2}\right)\right), \\
& g_{n} \rightarrow g \quad \text { in } \quad L^{2}\left(0, \infty ; H^{-2}\left(R^{2}\right)\right)
\end{aligned}
$$

as $n \rightarrow \infty$. We now define

$$
v_{0}^{n}(x, y, z)=\left\{\begin{array}{lr}
u_{0}^{n}(x, y, z) & \text { for } z \geq 0 \\
-u_{0}^{n}(x, y,-z) & \text { for } z<0
\end{array}\right.
$$




$$
\begin{gathered}
\tilde{f}_{n}(x, y, z)=\left\{\begin{array}{lr}
f_{n}(x, y, z) & \text { for } z \geq 0, \\
f_{n}(x, y,-z) & \text { for } z<0 ;
\end{array}\right. \\
\tilde{g}_{n}(x, y, z)= \begin{cases}g_{n}(x, y, z) & \text { for } z \geq 0, \\
-g_{n}(x, y,-z) & \text { for } z<0 ;\end{cases} \\
v_{1}^{n}(x, y, z)=\frac{d \tilde{f}_{n}}{d z}(x, y, z)+\tilde{g}_{n}(x, y, z) .
\end{gathered}
$$

Then, it is easy to see that as $n \rightarrow \infty$,

$$
v_{0}^{n} \text { converges in } L^{2}\left(R_{z} ; H^{-1}\left(R^{2}\right)\right)
$$

and

$$
v_{1}^{n} \text { converges in } \mathcal{X} \text {. }
$$

With $\left(u_{0}, u_{1}\right)$ replaced by $\left(v_{0}^{n}, v_{1}^{n}\right)$ in the Cauchy problem (1.1), we obtain a solution $v^{n} \in C\left([0, T] ; H^{m}\left(R^{3}\right)\right) \cap C^{1}\left([0, T] ; H^{m}\left(R^{3}\right)\right)$ for every $m \in R$. By Lemma 1.4, (1.28), and (1.29), we find that

$$
v^{n} \rightarrow v \quad \text { in } \quad C\left([0, T] ; L^{2}\left(R_{z} ; H^{-1}\left(R^{2}\right)\right)\right) \cap C^{1}([0, T] ; \mathcal{X})
$$

for some $v$. Since $v_{0}^{n}$ and $v_{1}^{n}$ are odd in $z$, it follows that $v^{n}$ is odd in $z$. So $v^{n}(t, x, y, 0)=0$. Since $v^{n}$ is a smooth solution, it is evident that the restriction of $v^{n}$ to $R_{+}^{3}$ satisfies $(1.11)$ with $\left(u_{0}, u_{1}\right)$ replaced by the restriction of $\left(v_{0}^{n}, v_{1}^{n}\right)$ to $R_{+}^{3}$. Now it follows from (1.30) that the restriction of $v$ to $R_{+}^{3}$ is a solution of (1.9). For the uniqueness of the solution, choose an arbitrary function $\psi(t, x, y, z) \in C_{0}^{\infty}\left((0, T) \times R_{+}^{3}\right)$, and find the solution of

$$
\left\{\begin{array}{l}
\phi_{t t}-\Delta \phi=\psi \quad \text { in } \quad(0, T) \times R_{+}^{3}, \\
\phi(t, x, y, 0)=0 \quad \text { in } \quad(0, T) \times R^{2}, \\
\phi(T, x, y, z)=0 \quad \text { in } \quad R_{+}^{3}, \\
\phi_{t}(T, x, y, z)=0 \quad \text { in } \quad R_{+}^{3} .
\end{array}\right.
$$

Then $\phi$ is eligible as a test function for (1.11). Let $w$ be the difference between two solutions satisfying (1.11). Then, it is apparent that

$$
\int_{0}^{\infty} \int_{0}^{T}\langle w, \psi\rangle_{3} d t d z=0
$$

and hence, $w \equiv 0$.

As mentioned earlier, some regularity conditions are necessary for the uniqueness of solution of (1.9). To see this, let us consider

$$
u=\delta(t-z),
$$

where $\delta$ is the Dirac delta measure. Then, $u$ is obviously a weak solution of the wave equation and satisfies the homogeneous initial-boundary conditions in the following sense.

$$
\lim _{t \rightarrow 0+}\langle u, \phi(x, y, z)\rangle=0,
$$




$$
\begin{aligned}
& \lim _{t \rightarrow 0+}\left\langle u_{t}, \phi(x, y, z)\right\rangle=0, \\
& \lim _{z \rightarrow 0+}\langle u, \psi(t, x, y)\rangle=0
\end{aligned}
$$

for every $\phi \in C_{0}^{\infty}\left(R_{+}^{3}\right)$ and $\psi \in C_{0}^{\infty}\left((0, T) \times R^{2}\right)$. The bracket denotes the duality pairing between distributions and test functions. In fact, this also satisfies the boundary condition $\mathcal{B} u=0$ at $z=0$ in the sense

$$
\lim _{z \rightarrow 0+}\langle\mathcal{B} u, \psi(t, x, y)\rangle=0
$$

for all $\psi \in C_{0}^{\infty}\left((0, T) \times R^{2}\right)$.

2. Proof of Theorem 0.1. We first outline the strategy of proof.

Step 1. For given $0<T<\infty, u_{0} \in H^{2}\left(R_{+}^{3}\right)$, and $u_{1} \in H^{1}\left(R_{+}^{3}\right)$ with $\frac{\partial}{\partial z} u_{0}(x, y, 0) \in H^{1}\left(R^{2}\right)$, we find $\tilde{u}_{0} \in H^{2}\left(R^{3}\right)$ and $\tilde{u}_{1} \in H^{1}\left(R^{3}\right)$ which satisfy the following conditions:

$$
\begin{gathered}
\tilde{u}_{0}=u_{0}, \quad \tilde{u}_{1}=u_{1} \quad \text { for } \quad z \geq 0 ; \\
\mathcal{B} \tilde{u}_{0} \in L^{2}\left(R_{z} ; H^{-1}\left(R^{2}\right)\right), \quad \mathcal{B} \tilde{u}_{1} \in \mathcal{X} ;
\end{gathered}
$$

there are sequences $\left\{w_{0}^{n}\right\}_{n=1}^{\infty},\left\{w_{1}^{n}\right\}_{n=1}^{\infty}$ in $C_{0}^{\infty}\left(R^{3}\right)$ such that

$$
w_{0}^{n}(x, y, z)=-w_{0}^{n}(x, y,-z), \quad w_{1}^{n}(x, y, z)=-w_{1}^{n}(x, y,-z) \quad \text { for }-2 T<z<2 T \text {. }
$$

Step 2. We proceed as in Proposition 1.6 with help of Lemmas 1.1 and 1.2. Find the solution $w^{n}$ of the Cauchy problem $(1.1)$ in $(0,2 T) \times R^{3}$ with the initial data $\left(w_{0}^{n}, w_{1}^{n}\right)$. Let $w$ be the limit of $\left\{w^{n}\right\}_{n=1}^{\infty}$. Then, the restriction of $w$ to $[0, T] \times R_{+}^{3}$ is a solution of (1.9) with initial data $\left(\mathcal{B} u_{0}, \mathcal{B} u_{1}\right)$.

Step 3. Find the solution $u$ of the Cauchy problem (1.1) in $(0,2 T) \times R^{3}$ with the initial data $\left(\tilde{u}_{0}, \tilde{u}_{1}\right)$. By the uniqueness of solution of $(1.1)$, we have $w \equiv \mathcal{B} u$, and the restriction of $u$ to $[0, T] \times R_{+}^{3}$ is a desired solution.

Step 4. Prove the uniqueness of solution of $(0.1)$ - (0.3).

One might wonder if we can bypass Step 1 and use Proposition 1.6 directly with initial data $\left(\mathcal{B} u_{0}, \mathcal{B} u_{1}\right)$ to satisfy the boundary condition $\mathcal{B} u=0$ at $z=0$. This is not possible since the solution obtained by Proposition 1.6 cannot be properly related to the solution required in Theorem 0.1. The trouble lies in inverting the operator $\mathcal{B}$. We now present the details of the proof. Obviously, only Step 1 and Step 4 require technical details. 
Construction of $\tilde{u}_{0}$ and $\tilde{u}_{1}$. Suppose that $u_{0} \in H^{2}\left(R_{+}^{3}\right)$ is given with $\frac{\partial}{\partial z} u_{0}(x, y, 0) \in$ $H^{1}\left(R^{2}\right)$. Let us consider the following initial value problem:

$$
\begin{gathered}
\frac{\partial^{2} \hat{\phi}}{\partial s^{2}}+|\xi|^{2} \frac{\partial \hat{\phi}}{\partial s}-|\xi|^{2} \hat{\phi}=2|\xi|^{2} \frac{\partial \hat{u}_{0}}{\partial s}(\xi, s), \quad s>0 \\
\hat{\phi}(\xi, 0)=2 \hat{u}_{0}(\xi, 0) \\
\frac{\partial \hat{\phi}}{\partial s}(\xi, 0)=0
\end{gathered}
$$

where $\xi=\left(\xi_{1}, \xi_{2}\right)$ is the dual variable of $(x, y)$ and $\hat{u}_{0}$ denotes the Fourier transform of $u_{0}$ with respect to $(x, y)$. Here $\xi$ is a parameter in this initial value problem, which is well posed for almost all $\xi \in R^{2}$. It is easy to find a solution $\hat{\phi}$ of $(2.4)-(2.6)$ by the variation of constants formula:

$$
\begin{aligned}
\hat{\phi}(\xi, s)= & 2 \int_{0}^{s} \frac{e^{\lambda_{1}(s-\eta)}}{\sqrt{|\xi|^{4}+4|\xi|^{2}}}|\xi|^{2} \frac{\partial \hat{u}_{0}}{\partial \eta}(\xi, \eta) d \eta \\
& -2 \int_{0}^{s} \frac{e^{\lambda_{2}(s-\eta)}}{\sqrt{|\xi|^{4}+4|\xi|^{2}}}|\xi|^{2} \frac{\partial \hat{u}_{0}}{\partial \eta}(\xi, \eta) d \eta \\
& -\frac{2\left(\lambda_{2} / \lambda_{1}\right) e^{\lambda_{1} s}}{1-\left(\lambda_{2} / \lambda_{1}\right)} \hat{u}_{0}(\xi, 0) \\
& +\frac{2 e^{\lambda_{2} s}}{1-\left(\lambda_{2} / \lambda_{1}\right)} \hat{u}_{0}(\xi, 0) \\
& =\hat{J}_{1}(\xi, s)+\hat{J}_{2}(\xi, s)+\hat{J}_{3}(\xi, s)+\hat{J}_{4}(\xi, s),
\end{aligned}
$$

where

$$
\left\{\begin{array}{l}
\lambda_{1}=\frac{1}{2}\left(-|\xi|^{2}+\sqrt{|\xi|^{4}+4|\xi|^{2}}\right) \\
\lambda_{2}=\frac{1}{2}\left(-|\xi|^{2}-\sqrt{|\xi|^{4}+4|\xi|^{2}}\right)
\end{array}\right.
$$

and

$$
\begin{gathered}
\hat{J}_{1}(\xi, s)=2 \int_{0}^{s} \frac{\lambda_{1} e^{\lambda_{1}(s-\eta)}}{\sqrt{|\xi|^{4}+4|\xi|^{2}}}|\xi|^{2} \hat{u}_{0}(\xi, \eta) d \eta \\
\hat{J}_{2}(\xi, s)=\frac{2|\xi|^{2}}{\sqrt{|\xi|^{4}+4|\xi|^{2}}} \hat{u}_{0}(\xi, s)+\frac{2 \lambda_{1} e^{\lambda_{1} s}}{\sqrt{|\xi|^{4}+4|\xi|^{2}}} \hat{u}_{0}(\xi, 0), \\
\hat{J}_{3}(\xi, s)=-2 \int_{0}^{s} \frac{e^{\lambda_{2}(s-\eta)}}{\sqrt{|\xi|^{4}+4|\xi|^{2}}}|\xi|^{2} \frac{\partial \hat{u}_{0}}{\partial \eta}(\xi, \eta) d \eta \\
\hat{J}_{4}(\xi, s)=\frac{2 e^{\lambda_{2} s}}{1-\left(\lambda_{2} / \lambda_{1}\right)} \hat{u}_{0}(\xi, 0) .
\end{gathered}
$$


We fix a function $\rho \in C_{0}^{\infty}(R)$ such that

$$
\rho(s)=1 \quad \text { for }|s| \leq 2 T
$$

and estimate each $\rho(s) \hat{J}_{i}(\xi, s)$. There is a positive constant $M$ such that for all $\xi \in R^{2}$,

$$
\begin{gathered}
0 \leq \lambda_{1} \leq M, \\
\left.\left|\lambda_{2}+\right| \xi\right|^{2} \mid \leq M .
\end{gathered}
$$

By means of (2.14) and the inequality

$$
\left\|(1+|\xi|)^{3 / 2} \hat{u}_{0}(\xi, 0)\right\|_{L^{2}\left(R^{2}\right)} \leq M\left\|u_{0}\right\|_{H^{2}\left(R_{+}^{3}\right)},
$$

it is evident that for $i=1,2$,

$$
\int_{0}^{\infty}\left(\left\|\frac{\partial^{2}}{\partial s^{2}}\left(\rho(s) \hat{J}_{i}(\xi, s)\right)\right\|_{L^{2}\left(R^{2}\right)}^{2}+\left\|\rho(s)\left(1+|\xi|^{2}\right) \hat{J}_{i}(\xi, s)\right\|_{L^{2}\left(R^{2}\right)}^{2}\right) d s \leq M\left\|u_{0}\right\|_{H^{2}\left(R_{+}^{3}\right)}^{2}
$$

for some positive constant $M$. Next we recall some basic estimates of solutions of the heat equation, which follow directly from energy estimates:

$$
\int_{0}^{\infty}\left\|e^{-|\xi|^{2} s}|\xi|^{2} \hat{f}(\xi)\right\|_{L^{2}\left(R^{2}\right)}^{2} d s \leq M\|f\|_{H^{1}\left(R^{2}\right)}^{2} \quad \text { for all } f \in H^{1}\left(R^{2}\right)
$$

and

$$
\int_{0}^{\infty}\left\|\int_{0}^{s} e^{-|\xi|^{2}(s-t)}|\xi|^{2} \hat{g}(\xi, t) d t\right\|_{L^{2}\left(R^{2}\right)}^{2} d s \leq M\|g\|_{L^{2}\left(R_{+}^{3}\right)}^{2} \quad \text { for all } g \in L^{2}\left(R_{+}^{3}\right)
$$

for some positive constant $M$. We can write $\lambda_{2}=-|\xi|^{2}+p(\xi)$ and

$$
\hat{J}_{4}(\xi, s)=\frac{2 e^{-|\xi|^{2} s}}{1-\left(\lambda_{2} / \lambda_{1}\right)} e^{p(\xi) s} \hat{u}_{0}(\xi, 0),
$$

where $p(\xi)$ is uniformly bounded, which follows from (2.15). By virtue of (2.14), (2.16), and (2.18), it is apparent that

$$
\int_{0}^{\infty}\left(\left\|\frac{\partial^{2}}{\partial s^{2}}\left(\rho(s) \hat{J}_{4}(\xi, s)\right)\right\|_{L^{2}\left(R^{2}\right)}^{2}+\left\|\left(1+|\xi|^{2}\right) \rho(s) \hat{J}_{4}(\xi, s)\right\|_{L^{2}\left(R^{2}\right)}^{2}\right) d s \leq M\left\|u_{0}\right\|_{H^{2}\left(R_{+}^{3}\right)}^{2} .
$$

We rewrite $\frac{\partial}{\partial s} \hat{J}_{3}$ as

$$
\begin{aligned}
\frac{\partial}{\partial s} \hat{J}_{3}(\xi, s)= & -2 \frac{|\xi|^{2} e^{\lambda_{2} s}}{\sqrt{|\xi|^{4}+4|\xi|^{2}}} \frac{\partial \hat{u}_{0}}{\partial s}(\xi, 0) \\
& -2 \int_{0}^{s} \frac{|\xi|^{2} e^{\lambda_{2}(s-\eta)}}{\sqrt{|\xi|^{4}+4|\xi|^{2}}} \frac{\partial^{2} \hat{u}_{0}}{\partial \eta^{2}}(\xi, \eta) d \eta, \\
& =\hat{I}_{1}(\xi, s)+\hat{I}_{2}(\xi, s),
\end{aligned}
$$


where $\hat{I}_{1}$ is the first term of the right-hand side, and $\hat{I}_{2}$ is the integral term. By the same argument as for $\hat{J}_{4}$, we have

$$
\int_{0}^{\infty}\left\|\frac{\partial\left(\rho \hat{I}_{1}\right)}{\partial s}(\xi, s)\right\|_{L^{2}\left(R^{2}\right)}^{2} d s \leq M\left\|\frac{\partial u_{0}}{\partial z}(x, y, 0)\right\|_{H^{1}\left(R^{2}\right)}^{2}
$$

for some positive constant $M . \hat{I}_{2}$ can be written as

$$
\hat{I}_{2}(\xi, s)=-2 e^{p(\xi) s} \int_{0}^{s} \frac{|\xi|^{2} e^{-|\xi|^{2}(s-\eta)}}{\sqrt{|\xi|^{4}+4|\xi|^{2}}} e^{-p(\xi) \eta} \frac{\partial^{2} \hat{u}_{0}}{\partial \eta^{2}}(\xi, \eta) d \eta
$$

Since $p(\xi)$ is uniformly bounded, we can derive by (2.19)

$$
\int_{0}^{\infty}\left\|\frac{\partial}{\partial s}\left(\rho(s) \hat{I}_{2}(\xi, s)\right)\right\|_{L^{2}\left(R^{2}\right)}^{2} d s \leq M\left\|\frac{\partial^{2} u_{0}}{\partial z^{2}}\right\|_{L^{2}\left(R_{+}^{3}\right)}^{2}
$$

for some positive constant $M$. By a similar argument, we can also estimate $\rho(s)(1+$ $\left.|\xi|^{2}\right) \hat{J}_{3}(\xi, s)$ and arrive at

$$
\begin{gathered}
\int_{0}^{\infty}\left(\left\|\frac{\partial^{2}}{\partial s^{2}}\left(\rho(s) \hat{J}_{3}(\xi, s)\right)\right\|_{L^{2}\left(R^{2}\right)}^{2}+\left\|\left(1+|\xi|^{2}\right) \rho(s) \hat{J}_{3}(\xi, s)\right\|_{L^{2}\left(R^{2}\right)}^{2}\right) d s \\
\leq M\left(\left\|u_{0}\right\|_{H^{2}\left(R_{+}^{3}\right)}^{2}+\left\|\frac{\partial u_{0}}{\partial z}(x, y, 0)\right\|_{H^{1}\left(R^{2}\right)}^{2}\right) .
\end{gathered}
$$

Combining (2.17), (2.21), and (2.26), we have

$$
\begin{gathered}
\int_{0}^{\infty}\left(\left\|\frac{\partial^{2}}{\partial s^{2}}(\rho(s) \hat{\phi}(\xi, s))\right\|_{L^{2}\left(R^{2}\right)}^{2}+\left\|\left(1+|\xi|^{2}\right) \rho(s) \hat{\phi}(\xi, s)\right\|_{L^{2}\left(R^{2}\right)}^{2}\right) d s \\
\leq M\left(\left\|u_{0}\right\|_{H^{2}\left(R_{+}^{3}\right)}^{2}+\left\|\frac{\partial u_{0}}{\partial z}(x, y, 0)\right\|_{H^{1}\left(R^{2}\right)}^{2}\right)
\end{gathered}
$$

where $M$ is a positive constant depending on $T$ through (2.13). We now define $\tilde{u}_{0}$ by

$$
\tilde{u}_{0}(x, y, z)=\left\{\begin{array}{l}
u_{0}(x, y, z) \quad \text { for } z \geq 0 \\
\rho(z) \phi(x, y,-z)-u_{0}(x, y,-z) \quad \text { for } z<0
\end{array}\right.
$$

where $\phi(x, y, s)$ is the Fourier inverse transform of $\hat{\phi}(\xi, s)$ above. Then, it follows that

$$
\left\{\begin{array}{l}
\lim _{z \rightarrow 0+} \tilde{u}_{0}(\cdot, z)=\lim _{z \rightarrow 0-} \tilde{u}_{0}(\cdot, z) \\
\lim _{z \rightarrow 0+} \frac{\partial}{\partial z} \tilde{u}_{0}(\cdot, z)=\lim _{z \rightarrow 0-} \frac{\partial}{\partial z} \tilde{u}_{0}(\cdot, z)
\end{array}\right.
$$

and consequently,

$$
\left\|\tilde{u}_{0}\right\|_{H^{2}\left(R^{3}\right)} \leq M\left(\left\|u_{0}\right\|_{H^{2}\left(R_{+}^{3}\right)}^{2}+\left\|\frac{\partial u_{0}}{\partial z}(x, y, 0)\right\|_{H^{1}\left(R^{2}\right)}^{2}\right),
$$

and

$$
\mathcal{B} \tilde{u}_{0} \in L^{2}\left(R_{z} ; H^{-1}\left(R^{2}\right)\right)
$$


It also follows that, for almost all $-2 T<z<2 T$,

$$
\left(\mathcal{B} \tilde{u}_{0}\right)(\cdot,-z)=-\left(\mathcal{B} \tilde{u}_{0}\right)(\cdot, z) \quad \text { in } H^{-1}\left(R^{2}\right) .
$$

Since $\mathcal{B} \tilde{u}_{0} \in L^{2}\left(R_{z} ; H^{-1}\left(R^{2}\right)\right)$, there are sequences $\left\{\zeta_{1}^{n}\right\}_{n=1}^{\infty}$ in $C_{0}^{\infty}\left((0,2 T) \times R^{2}\right)$, $\left\{\zeta_{2}^{n}\right\}_{n=1}^{\infty}$ in $C_{0}^{\infty}\left((2 T, \infty) \times R^{2}\right)$, and $\left\{\zeta_{3}^{n}\right\}_{n=1}^{\infty}$ in $C_{0}^{\infty}\left((-\infty,-2 T) \times R^{2}\right)$ such that as $n \rightarrow \infty$,

$$
\begin{array}{ll}
\zeta_{1}^{n} \rightarrow \chi_{1} \mathcal{B} \tilde{u}_{0} & \text { in } L^{2}\left(R_{z} ; H^{-1}\left(R^{2}\right)\right), \\
\zeta_{2}^{n} \rightarrow \chi_{2} \mathcal{B} \tilde{u}_{0} & \text { in } L^{2}\left(R_{z} ; H^{-1}\left(R^{2}\right)\right),
\end{array}
$$

and

$$
\zeta_{3}^{n} \rightarrow \chi_{3} \mathcal{B} \tilde{u}_{0} \quad \text { in } L^{2}\left(R_{z} ; H^{-1}\left(R^{2}\right)\right)
$$

where $\chi_{1}(z)$ is a characteristic function for the interval $[0,2 T], \chi_{2}(z)$ is for the interval $[2 T, \infty)$, and $\chi_{3}(z)$ is for $(-\infty,-2 T]$. We then set

$$
w_{0}^{n}(x, y, z)=\zeta_{1}^{n}(x, y, z)-\zeta_{1}^{n}(x, y,-z)+\zeta_{2}^{n}(x, y, z)+\zeta_{3}^{n}(x, y, z) .
$$

Then, all the conditions in (2.1)-(2.3) for $u_{0}$ are satisfied.

Next we suppose that $u_{1} \in H^{1}\left(R_{+}^{3}\right)$ is given. We consider (2.4)-(2.6) with $\hat{u}_{0}$ replaced by $\hat{u}_{1}$. Then, the solution $\hat{\psi}$ of $(2.4)-(2.6)$ is given by

$$
\hat{\psi}(\xi, s)=\hat{H}_{1}(\xi, s)+\hat{H}_{2}(\xi, s)+\hat{H}_{3}(\xi, s)+\hat{H}_{4}(\xi, s),
$$

where

$$
\begin{gathered}
\hat{H}_{1}(\xi, s)=2 \int_{0}^{s} \frac{\lambda_{1} e^{\lambda_{1}(s-\eta)}}{\sqrt{|\xi|^{4}+4|\xi|^{2}}}|\xi|^{2} \hat{u}_{1}(\xi, \eta) d \eta, \\
\hat{H}_{2}(\xi, s)=\frac{2|\xi|^{2}}{\sqrt{|\xi|^{4}+4|\xi|^{2}}} \hat{u}_{1}(\xi, s)+\frac{2 \lambda_{1} e^{\lambda_{1} s}}{\sqrt{|\xi|^{4}+4|\xi|^{2}}} \hat{u}_{1}(\xi, 0), \\
\hat{H}_{3}(\xi, s)=-2 \int_{0}^{s} \frac{e^{\lambda_{2}(s-\eta)}}{\sqrt{|\xi|^{4}+4|\xi|^{2}}}|\xi|^{2} \frac{\partial \hat{u}_{1}}{\partial \eta}(\xi, \eta) d \eta, \\
\hat{H}_{4}(\xi, s)=\frac{2 e^{\lambda_{2} s}}{1-\left(\lambda_{2} / \lambda_{1}\right)} \hat{u}_{1}(\xi, 0) .
\end{gathered}
$$

Since $u_{1} \in H^{1}\left(R_{+}^{3}\right)$ implies $u_{1}(x, y, 0) \in H^{1 / 2}\left(R^{2}\right)$, we can easily derive

$$
\int_{0}^{\infty}\left(\left\|\rho(s) \frac{\partial \hat{H}_{i}}{\partial s}(\xi, s)\right\|_{L^{2}\left(R^{2}\right)}^{2}+\left\|\rho(s)(1+|\xi|) \hat{H}_{i}(\xi, s)\right\|_{L^{2}\left(R^{2}\right)}^{2}\right) d s \leq M\left\|u_{1}\right\|_{H^{1}\left(R_{+}^{3}\right)}^{2},
$$


for $i=1,2,4$, where $\rho$ is the same as defined by (2.13). For the estimate of $\hat{H}_{3}$, we write

$$
\begin{aligned}
\frac{\partial \hat{H}_{3}}{\partial s}(\xi, s)= & -2 \frac{|\xi|^{2}}{\sqrt{|\xi|^{4}+4|\xi|^{2}}} \frac{\partial \hat{u}_{1}}{\partial s}(\xi, s) \\
& -2 \int_{0}^{s} \frac{\lambda_{2}|\xi|^{2} e^{\lambda_{2}(s-\eta)}}{\sqrt{|\xi|^{4}+4|\xi|^{2}}} \frac{\partial \hat{u}_{1}}{\partial \eta}(\xi, \eta) d \eta
\end{aligned}
$$

and, by means of (2.15) and (2.19), we find that

$$
\int_{0}^{\infty}\left\|\rho(s) \frac{\partial \hat{H}_{3}}{\partial s}(\xi, s)\right\|_{L^{2}\left(R^{2}\right)}^{2} d s \leq M\left\|u_{1}\right\|_{H^{1}\left(R_{+}^{3}\right)}^{2}
$$

for some positive constant $M$. It also holds that

$$
\int_{0}^{\infty}\left\|\rho(s)(1+|\xi|) \hat{H}_{3}(\xi, s)\right\|_{L^{2}\left(R^{2}\right)}^{2} d s \leq M\left\|u_{1}\right\|_{H^{1}\left(R_{+}^{3}\right)}^{2} .
$$

Let us define

$$
\tilde{u}_{1}(x, y, z)=\left\{\begin{array}{l}
u_{1}(x, y, z) \quad \text { for } z \geq 0 \\
\rho(z) \psi(x, y,-z)-u_{1}(x, y,-z) \quad \text { for } z<0 .
\end{array}\right.
$$

Then we have

$$
\lim _{z \rightarrow 0+} \tilde{u}_{1}(\cdot, z)=\lim _{z \rightarrow 0-} \tilde{u}_{1}(\cdot, z) \quad \text { in } H^{1 / 2}\left(R^{2}\right),
$$

which, together with (2.42), (2.44), and (2.45), yields

$$
\frac{\partial \tilde{u}_{1}}{\partial z} \in L^{2}\left(R^{3}\right)
$$

and

$$
\left\|\tilde{u}_{1}\right\|_{H^{1}\left(R^{3}\right)} \leq M\left\|u_{1}\right\|_{H^{1}\left(R_{+}^{3}\right)},
$$

for some positive constant $M$ depending on $T$ through (2.13). Next we choose a sequence $\left\{v^{m}\right\}_{m=1}^{\infty}$ in $H^{3}\left(R_{+}^{3}\right)$ such that

$$
v^{m} \rightarrow u_{1} \quad \text { in } H^{1}\left(R_{+}^{3}\right)
$$

as $m \rightarrow \infty$. Let $\hat{\psi}^{m}$ be the solution of (2.4)-(2.6) with $\hat{u}_{0}$ replaced by $\hat{v}^{m}$, and define

$$
\tilde{v}^{m}(x, y, z)=\left\{\begin{array}{l}
v^{m}(x, y, z) \quad \text { for } z \geq 0, \\
\rho(z) \psi^{m}(x, y,-z)-v^{m}(x, y,-z) \quad \text { for } z<0 .
\end{array}\right.
$$

Then, by virtue of (2.49), we find that

$$
\mathcal{B} \tilde{v}^{m} \rightarrow \mathcal{B} \tilde{u}_{1} \quad \text { in } \mathcal{X} .
$$


In the meantime, the result for $u_{0}$ implies that each $\mathcal{B} \tilde{v}^{m}$ can be approximated in $L^{2}\left(R_{z} ; H^{-1}\left(R^{2}\right)\right)$ by a sequence of functions in $C_{0}^{\infty}\left(R^{3}\right)$ which are odd in $z \in$ $(-2 T, 2 T)$. Hence, there is a sequence $\left\{w_{1}^{n}\right\}_{n=1}^{\infty}$ in $C_{0}^{\infty}\left(R^{3}\right)$ such that

$$
w_{1}^{n} \rightarrow \mathcal{B} \tilde{u}_{1} \quad \text { in } \mathcal{X}
$$

and

$$
w_{1}^{n}(x, y,-z)=-w_{1}^{n}(x, y,-z) \quad \text { for }-2 T<z<2 T .
$$

Obviously, all the conditions (2.1)-(2.3) for $u_{1}$ are satisfied.

Solutions of (1.1) with initial data $\left(\tilde{u}_{0}, \tilde{u}_{1}\right)$. Consider the Cauchy problem (1.1) with the initial data $\left(w_{0}^{n}, w_{1}^{n}\right)$. For each $n$, there is a unique solution $w^{n} \in C([0,2 T]$; $\left.H^{m}\left(R^{3}\right)\right) \cap C^{1}\left([0,2 T] ; H^{m-1}\left(R^{3}\right)\right)$ for every $m \in R$. By Lemma 1.2 and the condition (iii) in $(2.3), w^{n}(t, x, y, 0)=0$ for all $(t, x, y) \in[0, T] \times R^{2}$. Hence, the restriction of $w^{n}$ to $[0, T] \times R_{+}^{3}$ satisfies $(1.11)$ with $\left(u_{0}, u_{1}\right)$ replaced by the restriction of $\left(w_{0}^{n}, w_{1}^{n}\right)$ to $z>0$. Meanwhile, we have, by Lemma 1.4,

$$
w^{n} \rightarrow \mathcal{B} \tilde{u} \quad \text { in } C\left([0, T] ; L^{2}\left(R_{z} ; H^{-1}\left(R^{2}\right)\right)\right) \cap C^{1}([0, T] ; \mathcal{X}),
$$

where $\tilde{u}$ is the unique solution of (1.1) with the initial data $\left(\tilde{u}_{0}, \tilde{u}_{1}\right)$. Hence, the restriction of $\mathcal{B} \tilde{u}$ to $[0, T] \times R_{+}^{3}$ satisfies (1.11) with $\left(u_{0}, u_{1}\right)$ replaced by the restriction of $\left(\mathcal{B} u_{0}, \mathcal{B} u_{1}\right)$ to $z>0$. Consequently, the restriction of $\tilde{u}$ to $[0, T] \times R_{+}^{3}$ is a desired solution in Theorem 0.1 .

Uniqueness of the solution. Let $\Phi$ be the difference between two solutions in Theorem 0.1. Then, we have

$$
\begin{gathered}
\Phi \in C\left([0, T] ; H^{2}\left(R_{+}^{3}\right)\right) \cap C^{1}\left([0, T] ; H^{1}\left(R_{+}^{3}\right)\right), \\
\Phi(0, x, y, z)=0, \quad \Phi_{t}(0, x, y, z)=0 \quad \text { for }(x, y, z) \in R_{+}^{3}, \\
\Phi_{t t}-\Delta \Phi=0 \quad \text { in }(0, T) \times R_{+}^{3} .
\end{gathered}
$$

Since $\mathcal{B} \Phi$ is a solution of (1.9) with

$$
(\mathcal{B} \Phi)(0, \cdot)=0, \quad\left(\mathcal{B} \Phi_{t}\right)(0, \cdot)=0,
$$

it follows from Proposition 1.6 that

$$
\mathcal{B} \Phi \equiv 0 \quad \text { in }(0, T) \times R_{+}^{3} .
$$

By virtue of (2.57) and the domain of dependence of the solution, we also have

$$
\Phi=0 \quad \text { for } 0 \leq t<z .
$$

Since $\Phi \in C\left([0, T] ; H^{2}\left(R_{+}^{3}\right)\right)$, (2.60) implies that for each $t \in[0, T], \xi \in R^{2}$,

$$
\frac{\partial^{2} \hat{\Phi}}{\partial z^{2}}(t, \xi, z)-|\xi|^{2} \frac{\partial \hat{\Phi}}{\partial z}(t, \xi, z)-|\xi|^{2} \hat{\Phi}(t, \xi, z)=0
$$

holds in $\mathcal{D}^{\prime}((0, \infty))$. But it follows from $(2.61)$ that

$$
\hat{\Phi}(t, \xi, z)=0 \quad \text { for all } \xi \in R^{2}, 0 \leq t<z,
$$

which, together with (2.62), yields

$$
\hat{\Phi} \equiv 0 \quad \text { in } R_{+}^{3} .
$$

Hence, the solution of Theorem 0.1 is unique.

Finally, the estimate (0.10) follows from Lemma 1.1, (2.30), and (2.49). 
3. Proof of corollaries. For the proof of corollaries, it is enough to obtain necessary estimates of $\hat{\phi}(\xi, s)$ given by (2.7). We start from Corollary 0.2. Suppose $u_{0} \in H^{3}\left(R_{+}^{3}\right)$ and $\mathcal{B} u_{0}=0$ at $z=0$. By the same argument used before, it is easy to see for $i=1,2$,

$$
\int_{0}^{\infty}\left(\left\|\frac{\partial^{3}}{\partial s^{3}}\left(\rho(s) \hat{J}_{i}(\xi, s)\right)\right\|_{L^{2}\left(R^{2}\right)}^{2}+\left\|\rho(s)(1+|\xi|)^{3} \hat{J}_{i}(\xi, s)\right\|_{L^{2}\left(R^{2}\right)}^{2}\right) d s \leq M\left\|u_{0}\right\|_{H^{3}\left(R_{+}^{3}\right)}^{2},
$$

where $\rho(s)$ is the same as before. By means of (2.12) and (2.22), we write

$$
\begin{aligned}
\frac{\partial^{2} \hat{J}_{3}}{\partial s^{2}}(\xi, s) & +\frac{\partial^{2} \hat{J}_{4}}{\partial s^{2}}(\xi, s)=\frac{2 \lambda_{1} \lambda_{2}^{2} e^{\lambda_{2} s}}{\sqrt{|\xi|^{4}+4|\xi|^{2}}} \hat{u}_{0}(\xi, 0) \\
& -2 \frac{\lambda_{2}|\xi|^{2} e^{\lambda_{2} s}}{\sqrt{|\xi|^{4}+4|\xi|^{2}}} \frac{\partial \hat{u}_{0}}{\partial s}(\xi, 0)-2 \frac{|\xi|^{2} e^{\lambda_{2} s}}{\sqrt{|\xi|^{4}+4|\xi|^{2}}} \frac{\partial^{2} \hat{u}_{0}}{\partial s^{2}}(\xi, 0) \\
& -2 \int_{0}^{s} \frac{|\xi|^{2} e^{\lambda_{2}(s-\eta)}}{\sqrt{|\xi|^{4}+4|\xi|^{2}}} \frac{\partial^{3} \hat{u}_{0}}{\partial \eta^{3}}(\xi, \eta) d \eta .
\end{aligned}
$$

However, the first three terms in the right-hand side can be written as

$$
\begin{aligned}
-2 \frac{|\xi|^{2} e^{\lambda_{2} s}}{\sqrt{|\xi|^{4}+4|\xi|^{2}}} & \left(\frac{\partial^{2} \hat{u}_{0}}{\partial s^{2}}(\xi, 0)-|\xi|^{2} \frac{\partial \hat{u}_{0}}{\partial s}(\xi, 0)-|\xi|^{2} \hat{u}_{0}(\xi, 0)\right) \\
& +e^{\lambda_{2} s}\left(p_{1}(\xi) \frac{\partial \hat{u}_{0}}{\partial s}(\xi, 0)+p_{2}(\xi) \hat{u}_{0}(\xi, 0)\right)
\end{aligned}
$$

where $p_{1}(\xi)$ and $p_{2}(\xi)$ are uniformly bounded in $\xi$. Since $\mathcal{B} u_{0}=0$ at $z=0$, (3.2) is reduced to

$$
\begin{aligned}
\frac{\partial^{2} \hat{J}_{3}}{\partial s^{2}}(\xi, s)+\frac{\partial^{2} \hat{J}_{4}}{\partial s^{2}}(\xi, s)= & e^{\lambda_{2} s}\left(p_{1}(\xi) \frac{\partial \hat{u}_{0}}{\partial s}(\xi, 0)+p_{2}(\xi) \hat{u}_{0}(\xi, 0)\right) \\
& -2 \int_{0}^{s} \frac{|\xi|^{2} e^{\lambda_{2}(s-\eta)}}{\sqrt{|\xi|^{4}+4|\xi|^{2}}} \frac{\partial^{3} \hat{u}_{0}}{\partial \eta^{3}}(\xi, \eta) d \eta
\end{aligned}
$$

It now follows from (2.18) and (2.19) that

$$
\int_{0}^{\infty}\left\|\rho(s)\left(\frac{\partial^{3} \hat{J}_{3}}{\partial s^{3}}(\xi, s)+\frac{\partial^{3} \hat{J}_{4}}{\partial s^{3}}(\xi, s)\right)\right\|_{L^{2}\left(R^{2}\right)}^{2} d s \leq M\left\|u_{0}\right\|_{H^{3}\left(R_{+}^{3}\right)}^{2} .
$$

We can easily find that

$$
\int_{0}^{\infty}\left\|\rho(s)(1+|\xi|)^{3}\left(\hat{J}_{3}(\xi, s)+\hat{J}_{4}(\xi, s)\right)\right\|_{L^{2}\left(R^{2}\right)}^{2} d s \leq M\left\|u_{0}\right\|_{H^{3}\left(R_{+}^{3}\right)}^{2} .
$$

By virtue of (3.1) and (3.6), we can estimate $\phi$;

$$
\|\rho(z) \phi(x, y, z)\|_{H^{3}\left(R_{+}^{3}\right)} \leq M\left\|u_{0}\right\|_{H^{3}\left(R_{+}^{3}\right)},
$$

for some positive constant $M$, which depends on $T$ through (2.13). Recalling (2.4), (2.28), (2.29), and the boundary condition $\mathcal{B} u_{0}=0$ at $z=0$ we derive

$$
\lim _{z \rightarrow 0+} \frac{\partial^{2} \tilde{u}_{0}}{\partial z^{2}}(\cdot, z)=\lim _{z \rightarrow 0-} \frac{\partial^{2} \tilde{u}_{0}}{\partial z^{2}}(\cdot, z)
$$


which, combined with (3.7), yields

$$
\left\|\tilde{u}_{0}\right\|_{H^{3}\left(R^{3}\right)} \leq M\left\|u_{0}\right\|_{H^{3}\left(R_{+}^{3}\right)} .
$$

The estimate of $\tilde{u}_{1}$ defined by (2.46) follows directly from the estimate of $\tilde{u}_{0}$ in the previous section. This completes the proof of Corollary 0.2 .

For the proof of the remaining corollaries, we first observe the following fact.

Lemma 3.1. Let $\hat{\phi}$ be a solution of (2.4)-(2.6). Assume that $u_{0} \in H^{2 m+4}\left(R_{+}^{3}\right)$, and $\mathcal{B} u_{0}=0, \Delta \mathcal{B} u_{0}=0, \ldots, \Delta^{m} \mathcal{B} u_{0}=0$ at $z=0$, where $m$ is a nonnegative integer. Then, we have

$$
\begin{gathered}
\frac{\partial^{2 m+2} \hat{\phi}}{\partial s^{2 m+2}}(\xi, 0)=2 \frac{\partial^{2 m+2} \hat{u}_{0}}{\partial s^{2 m+2}}(\xi, 0) \quad \text { for almost all } \xi \in R^{2}, \\
\frac{\partial^{2 m+3} \hat{\phi}}{\partial s^{2 m+3}}(\xi, 0)=0 \quad \text { for almost all } \xi \in R^{2} .
\end{gathered}
$$

Proof. Suppose that $\mathcal{B} u_{0}=0$ at $z=0$. Then, it follows from (2.4)-(2.6) that

$$
\begin{aligned}
\frac{\partial^{2} \hat{\phi}}{\partial s^{2}}(\xi, 0)= & 2|\xi|^{2}\left(\hat{u}_{0}(\xi, 0)+\frac{\partial \hat{u}_{0}}{\partial s}(\xi, 0)\right) \\
& =2 \frac{\partial^{2} \hat{u}_{0}}{\partial s^{2}}(\xi, 0) \quad \text { for almost all } \xi \in R^{2}
\end{aligned}
$$

By differentiation of (2.4) in $s$, and using (3.12), we obtain

$$
\frac{\partial^{3} \hat{\phi}}{\partial s^{3}}(\xi, 0)=0 \quad \text { for almost all } \xi \in R^{2} .
$$

Suppose that Lemma 3.1 is true for $0 \leq m \leq k$. By differentiation of (2.4), we have

$$
\frac{\partial^{2 k+4} \hat{\phi}}{\partial s^{2 k+4}}+|\xi|^{2} \frac{\partial^{2 k+3} \hat{\phi}}{\partial s^{2 k+3}}-|\xi|^{2} \frac{\partial^{2 k+2} \hat{\phi}}{\partial s^{2 k+2}}=2|\xi|^{2} \frac{\partial^{2 k+3} \hat{u}_{0}}{\partial s^{2 k+3}},
$$

which, combined with (3.10) and (3.11) for $m=k$, yields

$$
\begin{aligned}
\frac{\partial^{2 k+4} \hat{\phi}}{\partial s^{2 k+4}}(\xi, 0)= & 2|\xi|^{2}\left(\frac{\partial^{2 k+3} \hat{u}_{0}}{\partial s^{2 k+3}}(\xi, 0)+\frac{\partial^{2 k+2} \hat{u}_{0}}{\partial s^{2 k+2}}(\xi, 0)\right) \\
& =2 \frac{\partial^{2 k+4} \hat{u}_{0}}{\partial s^{2 k+4}}(\xi, 0) \quad \text { for almost all } \xi \in R^{2} .
\end{aligned}
$$

Here we also used the fact that $\Delta^{m} \mathcal{B} u_{0}=0,0 \leq m \leq k+1$ at $z=0$ implies $\frac{\partial^{2 m}}{\partial z^{2 m}} \mathcal{B} u_{0}=0,0 \leq m \leq k+1$, at $z=0$. Now (3.10) is true for $m=k+1$. By differentiation of (3.14), we also get (3.11) for $m=k+1$. The proof is complete.

Under the same assumption as in Lemma 3.1, we consider the initial value problem for $0 \leq k \leq m+1$,

$$
\frac{\partial^{2} \hat{\psi}_{k}}{\partial s^{2}}+|\xi|^{2} \frac{\partial \hat{\psi}_{k}}{\partial s}-|\xi|^{2} \hat{\psi}_{k}=2|\xi|^{2} \frac{\partial^{2 k+1} \hat{u}_{0}}{\partial s^{2 k+1}}(\xi, s), \quad s>0
$$




$$
\begin{gathered}
\hat{\psi}_{k}(\xi, 0)=2 \frac{\partial^{2 k} \hat{u}_{0}}{\partial s^{2 k}}(\xi, 0), \\
\frac{\partial \hat{\psi}_{k}}{\partial s}(\xi, 0)=0
\end{gathered}
$$

By the above lemma, and the uniqueness of solution of (2.4)-(2.6), we find

$$
\hat{\psi}_{k} \equiv \frac{\partial^{2 k} \hat{\phi}}{\partial s^{2 k}} \quad \text { for } 0 \leq k \leq m+1 .
$$

Recalling (2.28), we derive

$$
\lim _{z \rightarrow 0+} \frac{\partial^{\nu}}{\partial z^{\nu}} \tilde{u}_{0}(\cdot, z)=\lim _{z \rightarrow 0-} \frac{\partial^{\nu}}{\partial z^{\nu}} \tilde{u}_{0}(\cdot, z), \quad 0 \leq \nu \leq 2 m+3 .
$$

Meanwhile, (2.27) yields

$$
\begin{gathered}
\int_{0}^{\infty}\left(\left\|\frac{\partial^{2}}{\partial s^{2}}\left(\rho(s) \hat{\psi}_{m+1}(\xi, s)\right)\right\|_{L^{2}\left(R^{2}\right)}^{2}+\left\|\left(1+|\xi|^{2}\right) \rho(s) \hat{\psi}_{m+1}(\xi, s)\right\|_{L^{2}\left(R^{2}\right)}^{2}\right) d s \\
\leq M\left(\left\|u_{0}\right\|_{H^{2 m+4}\left(R_{+}^{3}\right)}^{2}+\left\|\frac{\partial^{2 m+3} \hat{u}_{0}}{\partial z^{2 m+3}}(x, y, 0)\right\|_{H^{1}\left(R^{2}\right)}^{2}\right)
\end{gathered}
$$

By means of (2.18) and (2.19), we can obtain directly from (2.9)-(2.12)

$$
\int_{0}^{\infty}\left\|\rho(s)(1+|\xi|)^{2 m+4} \hat{J}_{i}(\xi, s)\right\|_{L^{2}\left(R^{2}\right)}^{2} d s \leq M\left\|u_{0}\right\|_{H^{2 m+4}\left(R_{+}^{3}\right)}^{2}, \quad i=1, \ldots, 4 .
$$

By (3.20)-(3.22), we arrive at

$$
\left\|\tilde{u}_{0}\right\|_{H^{2 m+4}\left(R^{3}\right)} \leq M\left(\left\|u_{0}\right\|_{H^{2 m+4}\left(R_{+}^{3}\right)}+\left\|\frac{\partial^{2 m+3} u_{0}}{\partial z^{2 m+3}}(x, y, 0)\right\|_{H^{1}\left(R^{2}\right)}\right) .
$$

When $u_{0} \in H^{2 m+3}\left(R_{+}^{3}\right)$, with $\mathcal{B} u_{0}=0, \ldots, \Delta^{m} \mathcal{B} u_{0}=0$ at $z=0$, we apply the estimate (3.7) to $\psi_{m}=\frac{\partial^{2 m} \phi}{\partial z^{2 m}}$ so that

$$
\left\|\tilde{u}_{0}\right\|_{H^{2 m+3}\left(R^{3}\right)} \leq M\left\|u_{0}\right\|_{H^{2 m+3}\left(R_{+}^{3}\right)} .
$$

Here, $M$ is a positive constant depending on $T$ and $m$. Since the esimates of $\tilde{u}_{1}$ are identical with those of $\tilde{u}_{0}$, the proof of Corollaries 0.3 and 0.4 is complete. Corollary 0.5 follows trivially from the previous ones.

Acknowledgment. I would like to thank Shuming Sun for a helpful discussion.

\section{REFERENCES}

[1] J. Bergh And J. Löfström, Interpolation Spaces, Springer-Verlag, Berlin, Heidelberg, New York, 1976.

[2] H. Bond, Waves on the surface of a compressible liquid, Proc. Cambridge Phil. Soc., 43 (1947), pp. 75-95. 
[3] G.F.D. Duff, Hyperbolic differential equations and waves, in Boundary Value Problems for Linear Evolution Partial Differential Equations, Proc. NATO Advanced Study Institute, H.G. Garnir, ed., D. Reidel, Dordrecht, Boston, 1976, pp. 27-155.

[4] L. Hörmander, Linear Partial Differential Operators, 4th ed., Springer-Verlag, Berlin, Heidelberg, New York, 1976.

[5] L.D. Landau And E.M. Lifshitz, Fluid Mechanics, Pergamon Press, London, Paris, Frankfurt, 1959.

[6] J.L. Lions and E. Magenes, Non-Homogeneous Boundary Value Problems and Applications, Vol. 1, Springer-Verlag, New York, Heidelberg, Berlin, 1972. 